\author{
Massimo Antonelli \\ Elie Azoulay \\ Marc Bonten \\ Jean Chastre \\ Giuseppe Citerio \\ Giorgio Conti \\ Daniel De Backer \\ François Lemaire \\ Herwig Gerlach \\ Johan Groeneveld \\ Goran Hedenstierna \\ Duncan Macrae \\ Jordi Mancebo \\ Salvatore M. Maggiore \\ Alexandre Mebazaa \\ Philipp Metnitz \\ Jerôme Pugin \\ Jan Wernerman \\ Haibo Zhang
}

Received: 18 February 2008

Accepted: 18 February 2008

Published online: 29 February 2008

(C) Springer-Verlag 2008

\section{Antonelli (৫)}

Università Cattolica del Sacro Cuore,

Department of Intensive Care and

Anesthesiology, Policlinico Universitario

A. Gemelli,

Largo A. Gemelli, 8, 00168 Rome, Italy

e-mail: m.antonelli@rm.unicatt.it

E. Azoulay

Saint Louis Hospital, Intensive Care

Medicine Unit,

Paris, France

\section{Bonten}

University Medical Center Utrecht,

Department of Medical Microbiology Julius

Center for Health Sciences \& Primary Care,

Utrecht, The Netherlands

\section{J. Chastre}

Hopital Pitié Salpétrière, Reanimation medicale,

Paris, France

G. Citerio

Ospedale S. Gerardo, Neurointensive Care

Unit,

Monza, Italy

\section{Year in review in Intensive Care Medicine, 2007. III. Ethics and legislation, health services research, pharmacology and toxicology, nutrition and paediatrics}

G. Conti $\cdot$ S. M. Maggiore

Università Cattolica del Sacro Cuore,

Department of Intensive Care and

Anesthesiology,

Rome, Italy

D. De Backer

Erasme Hospital, Service des Soins

Intensifs,

Brussels, Belgium

F. Lemaire

Henri Mondor Hospital, Intensive Care

Unit,

Creteil, France

H. Gerlach

Vivantes-Klinikum Neukoelln, Department of Anesthesiology,

Berlin, Germany

J. Groeneveld

VUMC, Intensive Care Medicine Unit,

Amsterdam, The Netherlands

\section{G. Hedenstierna}

Uppsala University, Department of Clinical Physiology,

Uppsala, Sweden

D. Macrae

Royal Brompton Hospital, Pediatric

Intensive Care Unit,

London, UK
J. Mancebo

Hospital Sant Pau, Intensive Care Medicine

Unit,

Barcelona, Spain

\section{A. Mebazaa}

Lariboisière Hospital, Department of Anesthesiology and Critical Care

Medicine,

Paris, France

P. Metnitz

University Hospital of Vienna, Department of Anesthesia and General Intensive Care Medicine,

Vienna, Austria

\section{J. Pugin}

University Hospital of Geneva, Intensive

Care Medicine Unit,

Geneva, Switzerland

\section{J. Wernerman}

Karolinska University Hospital, Departments of Anesthesiology and Intensive Care Medicine,

Stockholm, Sweden

H. Zhang

University of Toronto, Interdepartmental

Division of Critical Care Medicine,

Toronto, Canada 


\section{Ethics and legislation}

End of life

In July 2006, Intensive Care Medicine (ICM) launched a new series of articles on legislation on end of life in different countries, of course focused on intensive care unit (ICU) settings. The first paper came from India [1]. Then followed papers on the legal situation and actual court cases in Belgium, Germany, Italy, Brazil, Israel, the Netherlands, and the UK [2-8]. Others are still in the pipeline, namely from Austria and Spain. Looking at the whole series of published articles gives an interesting overview of the current situation in many countries, mostly - though not exclusively - in the Western world, which shows many similarities but also some important differences.

The first common finding is that justice is always at pains to deal with intensive care physicians when they go to court accused of murder or assassination, be it in Italy, Spain, Belgium, Canada, France or in the Netherlands [3, 7, 9-12]. The respect for refusal of care and for the patient's autonomy, even at the expense of his or her life, is commonplace, at least when the patient is conscious and able to speak for him- or herself, as in the case of Mrs. B., in the UK [2]. But, of course, ICU patients are rarely conscious at this stage, making it difficult to base end-of-life decisions on their explicit will. The writing of advance directives is encouraged in several countries, e g., France, Denmark, Germany, and the UK, and is currently being energetically discussed in Italy [8]. Euthanasia is considered a criminal offense everywhere but in Belgium and the Netherlands. Obviously, as stated by J.L. Vincent for Belgium [7] and E. Kompanje for the Netherlands [3], their national legislation on euthanasia rarely, if ever, applies to intensive care situations, providing one does not confuse withdrawal of hopeless care and euthanasia. In no other European country does legislation allow a physician to intentionally interrupt his patient's life. Futility - or therapeutic obstinacy (in French: "acharnement thérapeutique") - is also disapproved in all countries, but it is rarely written in laws. In many countries, specific recommendations as to ICU end-of-life practices have been drafted and disseminated by scientific societies. In his editorial opening the new ICM series, Jean-Michel Boles reminded his readers of the survey he carried out for the International Consensus Conference organized by the ESICM and the list of guidelines from national societies for intensive care that he drew up in 2003 [13]. In some countries professional organizations of physicians, as in Germany, Denmark and France, and/or national ethics committees, as in Italy, have also produced recommendations on end-of-life care, frequently more specific than the corresponding legislations.

The greatest difficulties and the maximum disharmony between national legislations is seen when the withdrawal of life support is at stake, and even more so when in the case incompetent patients.

The UK has probably the most ancient and elaborate corpus of case laws on end of life [2]. Back in 1993, it was judged lawful that a patient with a permanent vegetative state had his enteral nutrition catheter withdrawn (Airedale NHS Trust vs. Bland). The 2002 ruling in the Mrs. B. case, allowing the removal of a mechanical ventilator, is certainly a landmark in the field. The recognition of the "double effect" goes back to 1957 (R. vs. Bodkin Adams). Even the thorny issue of surrogacy (who should speak for the incapacitated patient?) has recently be settled by the Mental Incapacity Act of 2005. Germany similarly has a corpus of texts regulating physicians' practices for end-of-life care, both in legislation and in professional codes [4]. The "passive help to die" has been backed by the Supreme Court. However, more clarification is requested and, in 2006, the biannual German lawyers' congress (Deutscher Juristentag) recommended the legislative organs to "clarify present legislation and more distinctly exculpate passive and indirect help to die". Interestingly, family members are not legal representatives by nature; to become so, they have to be appointed by a judge. Due to the atrocities of the Nazi era, the German legislation is the most protective of all European regulations; not surprisingly, euthanasia is totally banned [14]. Since 1989, the withdrawal of the feeding tube of a patient in persistent vegetative state has been possible in the Netherlands [3], following a ruling of the Dutch Court of Appeal which considered artificial nutrition a treatment that could be interrupted if deemed futile. Also, in Dutch jurisprudence, administration of opiates at the end of life is good palliative care, and certainly not murder.

Israel enacted in December 2005 a law regulating the treatment of the dying patient [6], following 6 years of intense public debate. Interestingly, this is probably the only law anywhere hat defines precisely what is meant by the end of life: a dying patient "will die within six months" and the last two weeks are the "final stage". As explained by A. Steinberg and C. Sprung, the issue of withdrawing mechanical ventilation at the end of life was particularly difficult to solve for this democratic and Jewish state, torn between the two often opposing concepts of sanctity of life and respect for autonomy. In order to make acceptable the withdrawal of a mechanical ventilator, the Israeli law accepted that a built-in "timer" transforms it from a continuous form of life support to an intermittent one. Although this may seem somewhat Byzantine, the authors state that such an innovative approach "is psychologically helpful to health care providers". At odds with other national legislations, nutrition and fluids are considered basic needs and not a treatment, making their withdrawal impossible. In another article also published in ICM, Sprung et al. show the importance of religion, and more broadly cultural influences in general, on end-of-life practices all over Europe [15]. 
Dr. R.K. Mani explains in his article on end of life in India [1] how different can be the practices in two kind of ICUs, public and private. In public hospitals, "swamped" by too many patients, physicians rarely take end-of-life decisions, either because of the lack of a palliative care culture (bear in mind that it took decades in western ICUs to start considering the withdrawal and withholding of life-support practices) or for fear of legal proceedings; accordingly, "distraught families" take away their loved ones in order to have them die at home under the designation of "left against medical advice". In the private sector, the exorbitant cost of ICU care may run families into bankruptcy in the case of a prolonged stay, as the rate of limitation of life support is much less than in western ICUs. In Brazil [5], a similar dichotomy exists between ICUs run by the public and the private sector, with economic factors potentially influencing end-of-life decisions. In public hospitals with lack of ICU beds and rationing of resources, it is " . . feared that limitation of life saving therapies could be deliberately and inappropriately undertaken in underprivileged patients. Conversely, in private institutions where physicians are paid on a fee for service basis, it could potentially lead to the inappropriate continuation of treatment". There is in Brazil an urgent need for clarification and regulation of end-of-life practices, with a mobilization of practitioners, ethicists, philosophers, and lawyers, as well as clergymen, to call for a relevant legislation.

Two interesting case reports from Tunisia [16, 17] showed how one can reconcile ICU care and the wish of patients or their families to die "at home". At the request of families, and after detailed explanation, the dying patient is taken home in an ambulance with the medical team aboard. Then, once in bed with his/her family around, after any desired rituals have been performed, the resuscitation gear is removed.

Within Europe, Italy is currently the country where the situation is the most confused. Although many ICU physicians and professional organizations plead for clear legislation on end-of-life practices, there is currently no legal framework for matters as crucial as mechanical ventilation withdrawal and palliative sedation. Recent cases that have received huge media coverage have shaken Italian society. Bock et al. described in ICM [9] the Welby case, which demonstrated the complex interactions between professionals, justice, society, and religious authorities. This article gave rise to an abundant correspondence, now in press. Withdrawal of nutrition and hydration is another matter of dispute, in the wake of the Schiavo case in the USA, which provoked comments by the Italian national ethics committee and a very strong statement from the Vatican [18]. A major cause of difficulty for physicians is that there is no role for the family in representing the patient. The Italian parliament is currently drafting regulation on advance directives and surrogacy (the new concept of "amministratore di sostegno"). Another approach to solving these issues is to improve the end-of-life skills of ICU physicians, which could be achieved by modifying their curriculum [19].

A new frontier in the end-of-life realm, namely prehospital emergency care, has been reached by Drs Ferrand and Marty [20]. In a survey published in ICM in 2006, they showed that life-support withdrawal and withholding is common in France in the prehospital setting, as $76 \%$ of respondents could describe such a recent case. Families were not informed of the decision is nearly $40 \%$ of cases. It is obvious that the conditions the French law [11] imposes for allowing life support removal (information of family, inscription into medical files and collegiality of the decision) cannot be met in the context of emergency. Dr. Rocker, in a accompanying editorial, expressed his concern regarding "decisions made in haste..." [21].

\section{Legal and ethical issues in clinical research}

This series started in 2006 with an article by F. Lemaire on the 60th anniversary of the Nazi physicians' trial held at Nuremberg from December 1946 to July 1947 [22]. The message of the hearings, as revisited in the 1990s, goes much farther than the mere affirmation of the necessity of informed consent. It reminds us that clinical research is done on human beings for the benefit of future patients, the good of society at large, and is not intended to benefit those patients who are included in the trials. As such, patients are entitled to the protection of the law, which is the basic rationale for the regulation physicians/researchers are always complaining of.

In all member states of the European Union (EU) State Members, directive 2001/20 on the "approximation of the laws, regulations and administrative provisions of the Member States relating to the implementation of good clinical practice in the conduct of clinical trials on medicinal products for human use" has been translated into national legislations since 2004. It is certainly an important step forward, but a huge disparity between countries still remains. For intensive care physicians, the main issue is how to obtain informed consent from patients unable to grant it, due to emergency or alteration of cognitive functions, a vulnerable population requiring augmented protection. However, national laws and regulations are rarely able to combine respect for patients' autonomy and the possibility to conduct research in this context. In the UK, initially, in 2004, a rigid translation of directive 2001/20 yielded a ban on all types of emergency research, as no exception was foreseen for consent. A media campaign and efficient lobbying by intensive care physicians allowed a revision of the British legislation which was promulgated in 2005 [23]. Harvey et al., from the ICNARC group [24], used the data of an already published randomized trial, the PAC-Man trial, to assess the consent/assent granted during this trial including typical 
incompetent patients. The results were indeed heartening: although, as expected, only $2.6 \%$ of patients were able to provide consent, relatives' assent was obtained in $81 \%$ of cases. Even more interesting, retrospective consent was the rule, and refusal by the patients themselves, once they became conscious and were asked to consent, occurred in only $0.6 \%$ of cases, confirming a posteriori the proxy consent given at inclusion. Using hypothetical scenarios, Ciroldi et al. [25], from the French network "Famirea", assessed also the agreement between patients, family and physicians in 100 interviews. In one scenario (a minimal risk study), the discrepancy between patients and surrogates was $32 \%$, and with the greater-than-minimal scenario it reached $46 \%$. The difference between the two studies could result from the real-life situation in the British study and the simulation in the French one.

In the Netherlands, initially, following legislation in 1998 , the members of family authorized by law to give surrogate consent for research were restricted to the spouse or life companion, in contrast to other decisions commonly necessary in health care, which could be taken by all close members of family, parents and children. This was changed in 2006 and the number of possible surrogates was increased, improving the accrual in trials, as described by D.P. Veelo et al. [26]. Also in the Netherlands, Jansen et al. recently reported another pitfall in the research legislation, the interdiction by one research ethics committee (REC) of the use of research data obtained from incompetent persons, under the provision of the waiver of consent, if these persons die before any consent could be granted, either from them or from an authorized surrogate [27]. Similarly, in Norway, nearly all research implying the constitution of a collection of biological samples (biobank) ended after a Biobank Act was passed, which prohibited the conservation of such samples without the patient's direct consent [28]. After physicians and researchers, in particular the Norwegian Society of Anesthesiology, became vocal in denouncing such an unethical hurdle to research and public health, the law was finally altered and proxy consent approved [29].

Hicks et al. summarize in a recent paper [30] the missions, functioning and difficulties of data safety monitoring boards (DSMBs). These new bodies are of major importance in most ICU trials, at least in those dealing with potentially fatal diseases and/or vulnerable patients. The authors outline the composition of such committees (number of members, qualification), connections with investigators and sponsors, and frequency of meetings. Recent published sets of recommendations, from the EAMA and the FDA, are referenced. Special emphasis is put on the extreme difficulty ("committees operate in a realm of uncertainty") of monitoring a trial in severe diseases: any DSMB is always torn between the fear of not stopping a dangerous trial, and thus putting patients at risk, and the wish to let a trial continue to its end in order to yield the answer it is supposed to provide. Intensive care physicians have to get used to serving in DSMBs, as they are more and more frequently required by sponsors and administrative bodies ("competent authorities").

ICUs are "technology rich environments", as said by Scales and Laupacis [31] in their description of the Health Technology Assessment (HTA). It is remarkable, however, that most of the techniques and devices we use every day have never been rigorously tested. Are ICUs a "HTA free zone"? In their article, the authors describe the many factors that explain this situation, which comes as a surprise in the context of critical care medicine, usually so keen to evaluate its activity: some originate from the well-known peculiarities of intensive care (difficulties in defining outcomes, in isolating one element of progress amongst the frequent multi-component therapeutic strategies, in designing a control group or a "usual care group", etc.), others from the nature of the medical devices themselves. However, the authors strongly argue that no new technology should be introduced before a sound assessment of its cost-benefit ratio.

Limiting and assessing the risks of research in critical care patients remain the most difficult and frequently taxing issue. Weijer and Miller [32] analyze in depth the determination of the risk-benefit ratio of research protocols, from the specific point of view of vulnerable populations, as patients in our ICUs always are. The bottom line is the distinction they trace (= component analysis) between the therapeutic component of any protocol, which must fulfill the ethical requirement of "clinical equipoise", and its non-therapeutic component, which can be defined as what departs from "usual care" due to research. Figure 1 illuminates this central and operative concept. RECs should use such a diagram as an essential guideline when assessing research projects to be applied in ICUs.

\section{Health services research and ICU burden}

With increasing economic pressure, the cost-effectiveness of several diagnostic and therapeutic interventions have to be questioned, and eventually to be evaluated. Unnecessary costs arise when diagnostic tests are performed without a specific indication, and essential cost savings have been reported when such unnecessary tests are omitted. Routine chest radiographs (CXRs) are the subject of such a debate: in many institutions they are ordered routinely (on a daily basis), more or less as a screening tool. Other institutions order CXRs only for specific reasons. Graat et al. [33] conducted a prospective non-randomized study to evaluate the impact of changing from routine ordering to an on-demand CXR ordering system. Changing from one to the other system, they found that the number of CXRs per day was on average reduced from $1.1 \pm 0.3$ to $0.6 \pm 0.4$ $(p>0.005)$ per patient per day. Comparing both expected and unexpected findings, the authors concluded that diag- 
nostic value increased with the on-demand system: more unexpected findings were found in more patients $(9.5 \%$ vs. $6.4 \%$ ). Moreover, neither length of stay (LOS) in the ICU, nor readmission rate or mortality rates changed with introduction of the on-demand system. However, it must be emphasized that incidence and course of possible complications were not evaluated: for example, several abnormalities might have been missed or detected too late, with impact on morbidity and mortality. Using the general cohort's mortality and readmissions rate might thus not be accurate enough to decide whether this method is safe enough to be generally adopted.

The interest in the identification of at-risk patients outside the ICU is growing: physiological track-and-trigger warning systems have been used to identify patients on acute wards at risk of deterioration, as early as possible. Dr. Subbe and collaborators [34] evaluated three different warning systems: the medical emergency team call-out criteria (MET); the modified early warning score (MEWS); and the assessment score of sick-patient identification and step-up in treatment (ASSIST). Inter-rater and intra-rater reliability were assessed by intra-class correlation coefficients, kappa statistics and percentage agreement. Despite fair to moderate agreement on most physiological parameters, and fair agreement on the scores, there was significant variation in the reproducibility of different track-andtrigger warning systems, with better levels of agreement on triggers than on aggregate scores. The authors concluded that simpler systems had better reliability, with a detectable improvement of inter-rater agreement by using electronic calculations of scores.

Two studies were published in 2007 on the burden of the ICU during and after an ICU stay. In a prospective single-center study, Ouimet and colleagues estimated the incidence of delirium using a validated scale (the Intensive Care Delirium Screening Checklist for delirium) in 820 consecutive patients admitted to the ICU for more than $24 \mathrm{~h}$ [35]. Risk factors and outcomes of delirium were also determined. The incidence of delirium was $31.8 \%$ and was associated with higher ICU and hospital LOS and mortality. By multivariate analysis, delirium was associated with a history of hypertension and alcoholism, higher APACHE II score, and with clinical effects of sedative and analgesic drugs. Another study performed by Jones and colleagues explored the relationships between post-traumatic stress disorder (PTSD), patients' memories of the ICU, and sedation practices [36]. In this multicenter prospective observational study, patients' memories of ICU was undertaken at 1-2 weeks post ICU discharge in 238 critically ill survivors who received mechanical ventilation. PTSD-related symptoms were assessed 3 months after ICU discharge. The rate of PTSD was $9.2 \%$. Factors associated with the development of PTSD were recall of delusional memories, prolonged sedation, and physical restraint with no sedation. Preventive strategies were nicely pointed out by the authors.
Darmon et al. sought to determine the prognosis in cancer patients receiving renal replacement therapy [37]. Over a 42-month period, 94 cancer patients received dialysis for cute kidney injury. Hospital mortality was $51.1 \%$. Predictors of death in hospital included severity of associated organ dysfunctions and renal function deterioration after ICU admission. Strikingly, the presence of cancer had no detectable influence on hospital mortality after adjustment for gender, age, acute severity, and chronic health status.

More specifically, outcomes in 121 head and neck cancer patients with life-threatening complications were assessed by Soares et al. [38]. The main reasons for ICU admission were sepsis $(37 \%)$ and acute respiratory failure (20\%). Most patients received mechanical ventilation. Hospital and 6-month mortality rates were $56 \%$ and $72 \%$, respectively. Bad performance status, advanced disease, and the number of organ failures were associated with increased mortality in multivariate analysis. These two studies help to appraise the prognosis in cancer patients requiring intensive life support.

\section{Pharmacology and toxicology}

Cardiovascular drugs were listed as the fifth leading cause of poisoning death in 2001. Calcium channel blocker (CCB) overdosing is associated with severe cardiotoxicity and was reported to account for approximately $40 \%$ of all deaths due to cardiovascular drugs. CCBs inhibit the inward movement of calcium through the cell membrane to intracellular sites. This results in a slowing of conduction, bradycardia, followed by potential heart blocks and junctional and ventricular escape rhythms. Moreover, decreased contractility results in decreased cardiac output and blockade of calcium influx into the vascular smooth muscle cells in vasodilation. Moreover, CCBs have been associated with therapy-refractory profound hyperglycemia. Beside symptomatic therapy, hyperinsulinemia/euglycemia therapy (HIET), using high doses of infused insulin, has been recognized to be a therapeutic strategy. Greene et al. [39] report on the use of this therapy in seven patients with CCB poisoning, of whom six survived. Since in five of these patients other cardiovascular drugs were involved, the efficacy of the therapy cannot be judged by their results. However, the authors conclude that HIET generally constitutes a safe therapy which provides the highest benefit when administered early in the course of CCB poisoning.

Bonnet investigated glycine and ammonia plasma concentrations during a 72-h remifentanil infusion and the relationship between glycine concentration and remifentanil infusion rate [40]. Plasma glycine concentration was correlated with the remifentanil cumulative dose and the infusion rate and did not reach the toxic threshold. As glycine concentration was also correlated with creatinine clearance and because remifentanil was the only source of exoge- 
nous glycine, additional data are necessary to ascertain the safety of remifentanil infusion in ICU patients.

Life-threatening adverse drug reactions are often disregarded in the ICU setting, but might represent a major problem. In a prospective 6-month observational study, Grenouillet-Delacre et al. [41] found that preventable life-threatening adverse drug reactions were frequently involved in organ failure at admission to medical intensive care with poor identification. A panel of experts assessed putative serious adverse drug reaction(s) for each drug taken and each organ failure by using a standardized causality assessment method.

Of the 405 patients included, $111(27.4 \%)$ presented an adverse drug reaction leading to organ failure. In $48 \%$ of cases adverse drug reactions were preventable, 23\% were undiagnosed and 19\% contributed to death. Age over 75 years (OR 2.25; 95\% CI 1.15-4.38; $p=0.02$ ), receiving more than three drugs (OR 6.90; 95\% CI $1.44-33.00 ; p=0.02)$, and a diagnosis of hematological malignancy (OR 6.19; 95\% CI 2.07-18.53; $p=0.001$ ) were independently associated with serious adverse drug reactions.

\section{Enteral and parenteral nutrition}

Pluess et al. [42] demonstrated that two boluses of a fish oil-rich intravenous fat emulsion administered $48 \mathrm{~h}$ and $24 \mathrm{~h}$ before the endotoxin challenge attenuate the inflammatory response. The response was evaluated by physiological parameters and platelet membrane phospholipid composition. The clinical relevance of this mechanism remains to be elucidated.

A prior history of type II diabetes mellitus does not appear to be a further risk factor for gastric feed intolerance. Lam and colleagues [43] report a retrospective study where consecutive mechanically ventilated patients with at least $72 \mathrm{~h}$ of gastric feeding were identified. The occurrence of feed intolerance and the time taken to develop feed intolerance was not related to type II diabetes mellitus. On the other hand, gastric feed intolerance was associated with a greater use of morphine/midazolam and vasopressor support and a longer ICU LOS.

The implications of intra-abdominal hypertension have become obvious. Accurate measurements are, of course, crucial. Kimball et al. [44] evaluated the intraobserver and interobserver variability of bladder pressure measurements. The measurements were performed by the nursing staff, using a standardized device with a standardized clinical protocol. The accuracy and reliability were demonstrated by intraobserver and interobserver comparisons. Pearson's correlations for measured intra-abdominal pressure were 0.934 and 0.950 , respectively. In the case of intra-abdominal hypertension De Laet et al. [45] report that bolus administration of cisatracurium significantly decreases intra-abdominal pressure from $18 \mathrm{mmHg}$ (16-20) at baseline to $14 \mathrm{mmHg}(12-16)$ during $30 \mathrm{~min}$, with no effect on circulation or urinary production. The authors conclude that administration of cisatracurium can be used to temporarily reduce intra-abdominal pressure in patients with intra-abdominal hypertension.

\section{Miscellanea}

Is subclinical pancreatitis common in multiple organ failure? Denz et al. [46] reported daily serum lipase and pancreatitis-associated protein measurements in 130 consecutive critically ill patients staying in the ICU more than 5 days and without prior pancreatic disorder. A serum lipase level above 3 times the normal upper concentration was seen in 38 patients. Morphological alterations of the pancreas were found on a cCT scan in 7 out of 20 of these patients. The authors conclude that hyperlipasemia and elevated serum pancreatitis-associated protein levels is a common finding in critically ill patients indicative of pancreatic cellular stress, while morphological alterations of the pancreas are rare and of little clinical importance.

To assess the quality of bedside techniques to monitor bleeding, capillary HemoCue was compared with laboratory determination of blood hemoglobin in patients with gastrointestinal bleeding by Van de Louw et al. [47]. In $21 \%$ of cases the discrepancy between HemoCue and hemoglobin was greater than $1 \mathrm{~g} / \mathrm{dl}$. The authors conclude that therapeutic decisions based upon capillary HemoCue alone should be very cautious.

\section{Pediatrics}

Maintaining stable lung volume is crucial to effective pulmonary management during mechanical ventilation, whether for anesthesia or pulmonary support during critical illness. Several useful papers have recently added to our understanding of this area.

Jaecklin and colleagues [48] presented a simple study in which they investigated the capacity of six neonatal ventilators to maintain the set $\mathrm{V}(\mathrm{t})$ value in response to sudden changes in compliance or resistance when operating in volume-targeted mode. Their findings suggest that neonatal ventilators frequently deliver inaccurate $\mathrm{V}(\mathrm{t})$, and the devices were frequently found to deliver excessive $V(t)$ in response to sudden increases in compliance or decreases in resistance, potentially putting neonates ventilated in such modes at risk of barotrauma and volutrauma. Clinicians must remain vigilant when selecting equipment and seek to understand the performance characteristics of equipment in the context of any proposed use. Manufacturers' performance claims must be carefully scrutinized.

Copnell, Tingay and coworkers described in two papers their work on endotracheal suctioning. Copnell et al. [49] compared the effectiveness of open and closed 
tracheal suction in recovering secretions from both normal and saline-lavage induced lung injury during both conventional and high-frequency ventilation in an anesthetized rabbit model. Simulated thick and thin secretions, respectively iopamidol 2 and fluorescent mucin, were recovered equally efficiently by either closed or open suction from normal lungs, but closed suction was less effective than open suction at recovering both thick and thin secretions irrespective of ventilation mode. In a related paper Tingay et al. [50] described the effect of open tracheal suctioning in a group of seven newborn infants undergoing high-frequency oscillatory ventilation (HFOV). Lung volume was determined by inductive plethysmography immediately before and after open tracheal suctioning. The findings were perhaps not surprising, showing that lung volume was reduced (Delta $\mathrm{V}-13 \mathrm{ml} / \mathrm{kg}$, SD $4 \mathrm{ml} / \mathrm{kg}$ ) following tracheal suctioning. However, with restoration of HFOV with ventilator settings unchanged, recovery restoration of lung volume was rapid, with Delta V at $60 \mathrm{~s}$ after reconnection being only $1 \mathrm{ml} / \mathrm{kg}$ (SD $4 \mathrm{ml} / \mathrm{kg}$ ) below pre-suctioning baseline.

Duff and colleagues [51] reported the effect of sustained inflations delivered as $30-40 \mathrm{cmH}_{2} \mathrm{O}$ for $15-20 \mathrm{~s}$ as recruitment maneuver (RMs) in ventilated children aged 11 days to 14 years. Ninety-three RMs were undertaken in 32 children. No hemodynamic differences were noted between pre- and post-RM data. Oxygen requirements were significantly reduced for $6 \mathrm{~h}$ following RM. No air leaks were noted. Three of eight RMs in patients with altered intracranial compliance were associated with a spike in intracranial pressure. Von Ungern-Sternberg [52] reported an elegant study of the effect of two methods of prone positioning on functional residual capacity (FRC) and ventilation distribution determined by sulfur-hexafluoride multibreath washout. They reported that FRC and ventilation distribution were similar in supine and flat-prone positions, whilst parameters improved in the augmented prone position, in which gel pads were used to support the pelvis and upper thorax, resulting in less pressure on the lower thorax and abdomen. On the basis of this report, the practice recommendation to provide such support during prone ventilation in children on theoretical grounds is now supported by relevant evidence.

Two papers emphasized the differences between pediatric and adult respiratory intensive care. Santschi et al. [53] undertook a survey to characterize the physiological limits considered acceptable during weaning of children from mechanical ventilation. Ninety-seven intensivists from 49 pediatric ICUs (PICUs) in Canada, France, Switzerland and Belgium responded the survey based around weaning scenarios for children of varying ages. Although the median values for acceptable minimal and maximal respiratory rates, tidal volumes, and end-tidal $\mathrm{CO}_{2}$ were unremarkable, the survey highlighted a wide divergence in opinions on acceptable weaning limits. Two conclusions may be drawn from this paper. The authors themselves concluded that we need to better "organize and consolidate our thinking and then consider establishing guidelines". Others hold the view that weaning is more art than science, and that while minimum safety thresholds can be established, inter-patient variability in pediatric critical care make such physiologically based guidelines of dubious value. Bruijn and colleagues [54] presented a thoughtful review of their experience of acute lung injury (ALI) in children with Down syndrome admitted to PICU with an acute respiratory tract infection. In a retrospective case review, they identified ALI in 14/24 (58.3\%) of children with Down syndrome compared to 41/317 (12.9\%) in non-Down children. A similarly higher incidence for acute respiratory distress syndrome (ARDS) in Down syndrome was detected [11/24 (46\%) in Down children versus $21 / 317(7 \%)$ in non-Down children]. The explanation for this has yet to be elucidated, but the findings, if confirmed, suggest that intensivists should be especially alert for early signs of ALI and ARDS in this high risk population. Population-based epidemiological studies made possible by national data collection as occurs in the UK through the Paediatric Intensive Care Audit Network (PICANET; www.picanet.org.uk) should allow intensivists to confirm this finding and possibly identify other "high-risk" PICU subpopulations.

Inhaled nitric oxide (iNO) therapy has an important if limited role in the care of neonates with cardiorespiratory failure associated with pulmonary hypertension and some potential applications in some older children such as those with reactive pulmonary hypertension following cardiac surgery. One significant concern of many neonatologists and cardiac surgeons is the potential for iNO to increase the risk of bleeding. In a piglet model involving $30 \mathrm{~h}$ of iNO at $40 \mathrm{ppm}$, Albert et al. [55] showed that none of a broad range of coagulation parameters, including bleeding time and Adeplat-S, were affected and therefore the hypothesis that iNO increases the risk of bleeding was not supported, a reassuring finding, particularly for our neonatal and surgical colleagues.

Three papers published over the course of the year describe technical aspects of the application of mechanical ventilation in ventilated neonates. Fassassi [56] compared the performance of passive air conditioning using a heat and moisture exchanger (HME) with a heated humidifier (HH) in 1411 ventilated neonates. Higher absolute humidity was achieved using the $\mathrm{HH}$ system $(33.8 \pm 2.3 \mathrm{mg}$ $\left.\mathrm{H}_{2} \mathrm{O} / \mathrm{l}\right)$ than with an HME system $\left(32.4 \pm 2.8 \mathrm{mg} \mathrm{H}_{2} \mathrm{O} / \mathrm{l}\right)$. The performance of the HME system was better than the minimum performance required to condition inspired gases in adults, although the safety of such limits in neonates has not been confirmed. At the very least, data from this study can be used to support the use of HMEs during transfers within and between hospitals where $\mathrm{HH}$ are less easily deployed. Further work to ascertain the place of HMEs in neonatal critical care as an alternative to $\mathrm{HH}$ is required. Bollen and colleagues [57] 
undertook a meta-analysis of randomized controlled trials which compared high-frequency ventilation (HFV) with conventional ventilation (CV) in premature neonates with respiratory distress syndrome. Fifteen eligible trials were identified and variables were sought to explain the heterogeneity of reported outcomes. The relative risk of chronic lung disease was reduced when HFV was used in association with a low-volume ventilation strategy $(0.42,95 \%$ CI $0.06-2.48)$ and increased with high volume strategy $(2.02,95 \%$ CI $0.18-23.12)$. Other factors assessed, including the influence of gestational age, use of the Sensormedics 3100A oscillator, and prior time on $\mathrm{CV}$, had smaller effects. The important message from this report is not necessarily that of the favorable influence of a low-volume lung strategy in neonates, given the extensive evidence of its benefit in adult acute respiratory failure and logical pathophysiological argument. It is that after at least 15 trials, the $\mathrm{HFV}$ versus $\mathrm{CV}$ question has not been answered conclusively, as no single study was both adequately designed and adequately powered to clearly answer the HFV versus CV question. This should act as a wake-up call to future investigators to improve the design of comparative clinical trials and in particular to ensure that trial interventions are clearly understood, accurately described, and that any trial has adequate power to answer the research question posed.

Phenotypic susceptibility to infection poses important challenges to future patient-directed therapies in the prevention and treatment of sepsis and inflammation. Capoluongo and colleagues [58] investigated the genetic variants of mannose-binding lectin 2 and correlated these to pulmonary outcomes in a population of premature neonates. They determined that both the homozygous and heterozygous 550G mutation were associated with protective effects, and the heterozygous R52C mutation with unfavorable effects, in relation to pulmonary outcome. Michalek et al. [59] also investigated genetic variants in pediatric sepsis. In a prospective single-center study involving 345 consecutive PICU admissions with fever, SIRS sepsis, or MODS, these authors studied the clinical correlation of two gene polymorphisms of the BPI Taq gene. A statistically significant predisposition to Gram-negative sepsis in children carrying the BPI Taq GG variant together with the BPI $216 \mathrm{AG}$ or $-\mathrm{GG}$ variant was revealed $(p=0.008)$. These haplotypes were also associated with death due to sepsis or sepsis-related complications.

Pavcnik-Arnol et al. [60] investigated the utility of lipopolysaccharide-binding protein (LBP) and two markers from the same pathway, lipopolysaccharide and $\mathrm{CD} 14$, as a marker of sepsis in neonates and children. $\mathrm{C}$-reactive protein (CRP) and procalcitonin (PCT) were also measured. In summary, they determined that LBP concentration on the first day of suspected sepsis was a better marker of sepsis than lipopolysaccharide, soluble CD 14, PCT, and, in neonates younger than $48 \mathrm{~h}$, CRP. Rey and colleagues [61] analyzed the clinical value of PCT, CRP, and leukocyte count in the diagnosis of pediatric sepsis. In the study, which involved 94 children in a single PICU, patients were classified at the time of CRP and PCT measurement as "negative" SIRS, localized infection, sepsis, severe sepsis, and septic shock. The areas under the receiver operating curves for the diagnosis of septic patients were leukocyte count $0.53(95 \%$ CI 0.46-0.60), CRP 0.75 (0.70-0.80), PCT 0.91 (0.88-0.94). These findings are not new but are supportive of the use of PCT as a clinical tool in neonates and children. The authors' attempt to stratify patients on the basis of CRP and PCT values, although conceptually interesting, was perhaps less persuasive. In a small study population of neonates and children with organ dysfunction, Krueger et al. [62] determined that a significant increase in levels of serum vascular cell adhesion molecule-1 (VCAM-1) was associated with relative dysfunction of three or more organ systems. Furthermore, serum levels of the adhesion molecule E-selectin differed significantly between infectious and non-infectious causes of organ failure. Callister and colleagues [63] compared the levels of thioredoxin (Trx) TNF-alfa, and IL-1 in 35 children during the acute and recovery phases of meningococcal septic shock (MSS). Trx levels were significantly lower in children with MSS, during both the acute and the convalescent phases, than in controls $(n=25)$. The predictive or therapeutic potential of the detection of low Trx levels in children warrants further investigation. Staquet [64], rather than looking for markers of sepsis, evaluated the utility of a real-time polymerase-chain reaction assay for the detection of Neisseria meningitides $(\mathrm{Nm})$ in skin lesion biopsies in patients with purpura fulminans. PCR testing detected $\mathrm{Nm}$ in 34/34 (100\%) of patients tested compared with only 5/34 for skin culture. PCR was performed on both blood and skin biopsy material in 17 patients with a $\mathrm{Nm}$ detection rate of $100 \%$ for skin, but in only 10/17 (58.8\%) for blood. PCR techniques such as this can be used to provide confirmatory microbiological evidence of $\mathrm{Nm}$ or similar infection in situations where prior administration of antibiotics has eradicated live organisms. Whether such efforts lead to clinically important benefits, given the largely non-specific supportive nature of the ICU treatment of purpura fulminans, remains unproven.

Casartelli and colleagues [65] report a study describing the serum cortisol profile and adrenal response in children with septic shock and relate their findings to outcome. Adrenal function was evaluated on the basis of serum cortisol measured at the onset of shock and on response of serum cortisol $30 \mathrm{~min}$ after administration of $0.5 \mu \mathrm{g} / 1.73 \mathrm{~m}^{2}$. Adrenal insufficiency was detected in $17(77.3 \%)$ of the patients. All children who died had a baseline cortisol higher than $690 \mathrm{nmol} / \mathrm{l}$. A corticotrophin response of less than $250 \mathrm{nmol} / \mathrm{l}$ was associated with a $60 \%$ mortality. Adrenal insufficiency is a frequent finding in children with septic shock. The low-dose corticotrophin stimulation test may be a useful tool to 
determine between normal cortisol response to stress and relative adrenal insufficiency. The possible benefit of cortisol supplementation in children with sepsis and relative adrenal insufficiency, although recommended by some authorities, requires further investigation.

Nosocomial infections are increasingly recognized as having a significant adverse effect on the outcome of those undergoing critical care at all ages, as demonstrated in the observational study conducted by Garcia-Teresa and collaborators [66]. They reported 1 year's data from 20 Spanish PICUs. Data related to 1092 catheters were obtained. The rate of catheter-related bloodstream infection (CRBSI) detected was 6.4 per 1,000 catheter days (95\% CI 5.0-8.0). Multivariate regression analysis determined that lower patient weight, guidewire exchange, and $>12$ days dwell time were independent predictors of CRBSI. Factors which protected were use of povidone-iodine on hubs and use of porous versus impermeable dressing. Dubos and colleagues examined the applicability of the HELICS questionnaire and conducted a survey of European PICUs to determine the prevalence and nature of nosocomial infection surveillance programs in PICUs. Sixty-five PICUs from 23 countries responded. Wide variations in practice were noted with varying definitions in use for commonly reported infections. Adaption of standard protocols for the reporting of nosocomial infection data such as a pediatric adaption of the HELICS protocol would assist European PICUs in establishing comparative benchmarks from which better clinical guidelines to reduce nosocomial infection rates could be developed.

Hatherill and colleagues [67] explored the relationship between blood lactate, pyruvate metabolic acidosis, and morbidity in a prospective observational study in children following cardiac surgery. Determined on PICU admission blood samples, metabolic acidosis occurred in $74 \%$ of children, hyperlactatemia ( $>2 \mathrm{mmol} / \mathrm{l}$ ) in $42 \%$, and raised lactate:pyruvate ratio in $45 \%$. Neither standard bicarbonate nor lactate:pyruvate ratio were independently associated with prolongation of PICU support, in contrast to lactate, which was associated with 0.42 days stay for each $1 \mathrm{mmol}$ increase in lactate above $2 \mathrm{mmol} / \mathrm{l}$.

The hypoplastic left heart syndrome and its variants present considerable challenges to pediatric cardiac surgeons and intensivists. Its initial palliation with the stage- 1 Norwood procedure is associated with significant mortality and morbidity even in experienced "high-volume" programs. Di Filippo and colleagues [68] describe their early experiences with a modification of the "classical" Norwood procedure, which employs a Blalock-Taussig shunt (BTS) to secure pulmonary blood flow with a recently promoted alternative, using a right ventricle to pulmonary artery (RVPA) conduit. Thirteen of 33 babies failed the procedure (defined as death or transplantation), $10(52 \%)$ in the BTS group and $3(21.4 \%)$ in the RVPA group. The authors speculate that this improvement in survival with the RVPA procedure may be due to the avoidance of excessive pulmonary blood flow and higher diastolic blood pressures achieved. Currently a large randomized trial comparing the two procedures is in progress (NCT00115934; www.clinicaltrials.gov), and its results, which will include assessment of longer-term effects, are eagerly awaited. In the current era, mortality rates from cardiac surgery continue to decline and are below $2 \%$ in many centers. Bontadelli and colleagues [69] report using the low-molecular-weight heparin, enoxaparin (EX), to treat a series of 32 infants with congenital heart disease who developed catheter-related arterial thrombi. Anticoagulant effect was monitored by measuring anti-factor Xa levels. Newborns required higher doses of EX (mean $1.62 \mathrm{mg} / \mathrm{kg}$ ), compared to $1.12 \mathrm{mg} / \mathrm{kg}$ per dose in infants aged 2-12 months. Therapeutic anti-Xa levels were achieved in all children. Bleeding complications occurred in only one child. EX appeared in this small series to be an alternative to the previously recommended therapy of unfractionated heparin.

Phrenic nerve injury and consequent hemi-diaphragm palsy is a recognized complication of cardiac surgery and can result in respiratory failure and ventilator dependence immediately following injury. It may be managed conservatively, particularly in older children, or with diaphragmatic plication, which is more commonly required in neonates and infants to facilitate weaning from respiratory support. Although the short-term outcomes of these treatment strategies have been described, longer-term outcomes have not, a deficiency addressed by Lemmer and colleagues [70]. They assessed a group of 27 patients at a median of 5.3 (range 3.0-12.6) years following cardiac surgery. Of particular interest in this series were the results of respiratory function tests performed on 15 patients and 39 case-matched controls with normal diaphragm function. Vital capacity (VC) and FEV1 were lower among patients with diaphragmatic palsy than in controls: VC $54.3 \%$ vs. $76.4 \%, p<0.001$; FEV1 $58.4 \%$ vs. $86.2 \%, p<0.001$. Patients in the diaphragm group also had a lower exercise capacity than those in the control group ( 24.5 vs. $31.3 \mathrm{ml} / \mathrm{kg} / \mathrm{min}, p=0.03$ ). These are important findings suggesting that the effect of diaphragm palsy remain significant beyond the short-term recovery phase. Plication may be beneficial, even in those patients who separate from respiratory support without plication, to improve longer-term respiratory performance. Longer-term follow-up studies are warranted.

Shankar and colleagues [71] published a preliminary report into the use of the use of a new long-acting insulin analog, insulin glarginine, in the treatment of diabetic ketoacidosis (DKA) in children. The authors propose that introducing a long-acting insulin to the treatment of DKA could lead to a quicker and smoother transition from continuous intravenous insulin to regular subcutaneous dosage. The report describes the treatment of 12 children who received 0.3 units $/ \mathrm{kg}$ of subcutaneous insulin glarginine (IG) with low-dose intravenous insulin (initial dose 
0.05-0.1 units $/ \mathrm{kg} / \mathrm{h})$. Comparisons are presented against a group of 59 children who received standard treatment alone over the same period. The mean time for acidosis correction and the insulin infusion time were both shorter in the IG group, with a trend towards shorter hospital stay favoring the IG group. This is clearly an encouraging finding and strongly suggests that a randomized controlled trial is needed to assess the new therapy.

Mehta and colleagues [72] undertook an ex-vivo study of the sequestration of a range of drugs to extracorporeal membrane oxygenation circuits containing a silicone membrane oxygenator. At the end of a $24-\mathrm{h}$ test period, varying percentages of the range of drugs tested were lost, ranging from $15.4 \%$ of ampicillin to $100 \%$ of fentanyl being lost from blood-primed circuits. It has long been understood that materials used in extracorporeal circuits have the potential to absorb drugs and that lipid-soluble drugs such as fentanyl are more avidly taken up by most plastic materials than more polar drugs such as penicillin-derived antimicrobials. In addition, some drugs such as catecholamines are known to spontaneously degrade with time under certain conditions. Although the levels of lipid-soluble drugs fall rapidly in ex-vivo circuits, the high volume of distribution of lipid-soluble drugs in the body leads to less pronounced in-vivo effects, counterbalancing to some extent the influence the "sink effect" of the extracorporeal circuit. This report did not address the interaction with the patient, and therefore the changes noted are likely to be more marked than in clinical reality. Nevertheless, the important message for clinicians is that dose adjustments may be necessary in vivo, particularly for those drugs which are avidly bound by circuit components.

The study reported by Kneyber and colleagues [73] is of interest as its publication occurred almost simultaneously with that of the TRIPICU study, a multicenter trial of conservative versus standard red cell transfusion thresholds in children. The Kneyber paper, a retrospective epidemiological cohort study, reports that red cell transfusion appears to be associated with increased mortality, prolonged duration of mechanical ventilation, and prolonged PICU stay. This finding is congruent with a number of reports from adult critical care but was not supported by the TRIPICU study, which did not demonstrate excessive mortality or morbidity in the "transfused" group.
Two papers addressed the important issue of severity of illness and mortality prediction scoring. Kuzniewicz et al. [74] evaluated whether physiological trends and interaction terms could enhance the predictive ability in a variant of the Richardson score. The authors used sophisticated modeling techniques applied to data from a cohort of 2,222 newborns of 34 weeks gestation or more with respiratory distress. They concluded that incorporating trend and interaction terms into severity scores can enhance predictive ability, and that in the current digital era such inputs are both feasible and desirable. Wolfler and colleagues [75], on behalf of the Italian Paediatric Sepsis Study, evaluated the performance of the Pediatric Index of Mortality 2 score (PIM2) in Italian pediatric intensive care units. Overall, the score performed well, further enhancing the reputation of this easy-to-collect mortality prediction score.

Schieveld and colleagues [76] presented a report on the incidence of delirium during critical illness in children. They conclude that whilst under-reported and under-represented in the published literature, the incidence of delirium in PICU patients is not negligible and deserves more effort to better understand its phenomenology, risk factors and treatment. Drug tolerance and, in particular, withdrawal syndromes after periods of escalating analgeso-sedative administration are of great concern to pediatric intensivists and also parents. Cheung and coworkers [77] set out to explore the hypothesis that selective antagonism of excitatory opioid receptor functions with low concentrations of opioid antagonists might increase the efficacy of opioid analgesia and attenuate opioid tolerance. Randomized trials in adult patients report that low-dose naloxone reduces side effects and opioid requirements without increasing postoperative pain scores. In this small preliminary study in which low-dose naloxone was infused in 14 PICU children requiring infusions of opioids, data from the intervention group were compared to those from 12 case-matched controls. The two groups did not differ in terms of pain scores, sedation scores, or opioids' side effects. The authors concluded by acknowledging the limitations of their case-control methodology and suggesting that relatively large randomized controlled trials would be required to explore this question further.

\section{References}

1. Mani RK (2006) End-of-life care in India. Intensive Care Med 32:1066-1068

2. Bell D (2007) The legal framework for end of life care: a United Kingdom perspective. Intensive Care Med 33:158-162
3. Kompanje EJ (2006) Care for the dying in intensive care in The Netherlands. Intensive Care Med 32:2067-2069

4. Michalsen A (2007) Care for dying patients - German legislation. Intensive Care Med 33:1823-1826
5. Soares M, Terzi RG, Piva JP (2007) End-of-life care in Brazil. Intensive Care Med 33:1014-1017

6. Steinberg A, Sprung CL (2006) The dying patient: new Israeli legislation. Intensive Care Med 32:1234-1237 
7. Vincent JL (2006) End-of-life practice in Belgium and the new euthanasia law. Intensive Care Med 32:1908-1911

8. Zamperetti N, Proietti R (2006) End of life in the ICU: laws, rules and practices: the situation in Italy. Intensive Care Med 32:1620-1622

9. Bock M, Ciarrocchi V, Wiedermann CJ (2007) Case involving end-of-life decision issues in Italy. Intensive Care Med 33:1041-1042

10. Del Nogal Saez F (2006) Opiates at the end of life in an emergency department in Spain: euthanasia or good clinical practice? Intensive Care Med 32:1086-1087

11. Lemaire FJ (2004) A law for end of life care in France? Intensive Care Med 30:2120

12. Rocker G (2003) End of life care in Canada after a murder charge in an ICU. Intensive Care Med 29:2336-2337

13. Boles JM (2006) End of life in the intensive care unit: from practice to law. What do the lawmakers tell the caregivers? A new series in Intensive Care Medicine. Intensive Care Med 32:955-957

14. Michalsen A, Reinhart K (2006) "Euthanasia": a confusing term, abused under the Nazi regime and misused in present end-of-life debate. Intensive Care Med 32:1304-1310

15. Sprung CL, Maia $\mathrm{P}$, Bulow $\mathrm{HH}$, Ricou B, Armaganidis A, Baras M, Wennberg E, Reinhart K, Cohen SL, Fries DR, Nakos G, Thijs LG (2007) The importance of religious affiliation and culture on end-of-life decisions in European intensive care units. Intensive Care Med 33:1732-1739

16. Boussarsar M, Bouchoucha S (2006) Dying at home: cultural and religious preferences. Intensive Care Med 32:1917-1918

17. Kallel H, Dammak H, Bahloul M, Ben Hamida C, Chelly H, Rekik N, Bouaziz M (2006) A good death: another break in the wall. Intensive Care Med 32:1915-1916

18. Zamperetti N, Latronico N (2006) Nutrition and hydration of patients in vegetative state: a statement of the Italian National Committee for Bioethics. Intensive Care Med 32:750-751

19. Giannini A, Pinto AM, Rossetti G, Prandi E, Tiziano D, Brahe C, Nardocci N (2006) Respiratory failure in infants due to spinal muscular atrophy with respiratory distress type 1 . Intensive Care Med 32:1851-1855

20. Ferrand E, Marty J (2006) Prehospital withholding and withdrawal of life-sustaining treatments. The French LATASAMU survey. Intensive Care Med 32:1498-1505
21. Rocker G (2006) Life-support limitation in the pre-hospital setting. Intensive Care Med 32:1464-1466

22. Lemaire F (2006) The Nuremberg doctors' trial: the 60th anniversary. Intensive Care Med 32:2049-2052

23. Coats TJ, Shakur H (2005) Consent in emergency research: new regulations. Emerg Med J 22:683-685

24. Harvey SE, Elbourne D, Ashcroft J, Jones CM, Rowan K (2006) Informed consent in clinical trials in critical care: experience from the PAC-Man Study. Intensive Care Med 32:2020-2025

25. Ciroldi M, Cariou A, Adrie C, Annane D, Castelain V, Cohen Y, Delahaye A, Joly LM, Galliot R, Garrouste-Orgeas M, Papazian L, Michel F, Barnes NK, Schlemmer B, Pochard F, Azoulay E (2007) Ability of family members to predict patient's consent to critical care research. Intensive Care Med 33:807-813

26. Veelo DP, Spronk PE, Kuiper MA, Korevaar JC, van der Voort PH, Schultz MJ (2006) A change in the Dutch Directive on Medical Research Involving Human Subjects strongly increases the number of eligible intensive care patients: an observational study. Intensive Care Med 32:1845-1850

27. Jansen TC, Kompanje EJ, Druml C, Menon DK, Wiedermann CJ, Bakker J (2007) Deferred consent in emergency intensive care research: what if the patient dies early? Use the data or not? Intensive Care Med 33:894-900

28. Klepstad P, Dale O (2006) Further restrictions for ICU research. Intensive Care Med 32:175

29. Klepstad P, Guttormsen AB, Dale O (2007) Common sense won: engagement from experts repealed Norwegian restrictions for ICU research. Intensive Care Med 33:1827-1828

30. Hicks LK, Laupacis A, Slutsky AS (2007) A primer on data safety monitoring boards: mission, methods, and controversies. Intensive Care Med 33:1815-1818

31. Scales DC, Laupacis A (2007) Health technology assessment in critical care. Intensive Care Med 33:2183-2191

32. Weijer C, Miller PB (2007) Evaluating benefits and harms in intensive care research. Intensive Care Med 33:1819-1822

33. Graat ME, Kroner A, Spronk PE, Korevaar JC, Stoker J, Vroom MB, Schultz MJ (2007) Elimination of daily routine chest radiographs in a mixed medical-surgical intensive care unit. Intensive Care Med 33:639-644

34. Subbe CP, Gao H, Harrison DA (2007) Reproducibility of physiological track-and-trigger warning systems for identifying at-risk patients on the ward. Intensive Care Med 33:619-624
35. Ouimet $\mathrm{S}$, Kavanagh BP, Gottfried SB, Skrobik Y (2007) Incidence, risk factors and consequences of ICU delirium. Intensive Care Med 33:66-73

36. Jones C, Backman C, Capuzzo M, Flaatten H, Rylander C, Griffiths RD (2007) Precipitants of post-traumatic stress disorder following intensive care: a hypothesis generating study of diversity in care. Intensive Care Med 33:978-985

37. Darmon M, Thiery G, Ciroldi M, Porcher R, Schlemmer B, Azoulay E (2007) Should dialysis be offered to cancer patients with acute kidney injury? Intensive Care Med 33:765-772

38. Soares M, Salluh JI, Toscano L, Dias FL (2007) Outcomes and prognostic factors in patients with head and neck cancer and severe acute illnesses. Intensive Care Med 33:2009-2013

39. Greene SL, Gawarammana I, Wood DM, Jones AL, Dargan PI (2007) Relative safety of hyperinsulinaemia/euglycaemia therapy in the management of calcium channel blocker overdose: a prospective observational study. Intensive Care Med 33:2019-2024

40. Bonnet MP, Minville V, Asehnoune K, Bridoux D, Poggi-Bach J, Duranteau J, Benhamou D (2007) Glycine and ammonia plasma concentrations during sedation with remifentanil in critically ill patients. Intensive Care Med 33:1179-1182

41. Grenouillet-Delacre M, Verdoux H, Moore N, Haramburu F, MiremontSalame G, Etienne G, Robinson P, Gruson D, Hilbert G, Gabinski C, Begaud B, Molimard M (2007) Lifethreatening adverse drug reactions at admission to medical intensive care: a prospective study in a teaching hospital. Intensive Care Med 33:2150-2157

42. Pluess TT, Hayoz D, Berger MM, Tappy L, Revelly JP, Michaeli B, Carpentier YA, Chiolero RL (2007) Intravenous fish oil blunts the physiological response to endotoxin in healthy subjects. Intensive Care Med 33:789-797

43. Lam SW, Nguyen NQ, Ching K, Chapman M, Fraser RJ, Holloway RH (2007) Gastric feed intolerance is not increased in critically ill patients with type II diabetes mellitus. Intensive Care Med 33:1740-1745

44. Kimball EJ, Mone MC, Wolfe TR, Baraghoshi GK, Alder SC (2007) Reproducibility of bladder pressure measurements in critically ill patients. Intensive Care Med 33:1195-1198

45. De Laet I, Hoste E, Verholen E, De Waele JJ (2007) The effect of neuromuscular blockers in patients with intra-abdominal hypertension. Intensive Care Med 33:1811-1814 
46. Denz C, Siegel L, Lehmann KJ, Dagorn JC, Fiedler F (2007) Is hyperlipasemia in critically ill patients of clinical importance? An observational $\mathrm{CT}$ study. Intensive Care Med 33:1633-1636

47. Van de Louw A, Lasserre N, Drouhin F, Thierry S, Lecuyer L, Caen D, Tenaillon A (2007) Reliability of HemoCue in patients with gastrointestinal bleeding. Intensive Care Med 33:355-358

48. Jaecklin T, Morel DR, Rimensberger PC (2007) Volume-targeted modes of modern neonatal ventilators: how stable is the delivered tidal volume? Intensive Care Med 33:326-335

49. Copnell B, Tingay DG, Kiraly NJ, Sourial M, Gordon MJ, Mills JF, Morley CJ, Dargaville PA (2007) A comparison of the effectiveness of open and closed endotracheal suction. Intensive Care Med 33:1655-1662

50. Tingay DG, Copnell B, Mills JF, Morley CJ, Dargaville PA (2007) Effects of open endotracheal suction on lung volume in infants receiving HFOV. Intensive Care Med 33:689-693

51. Duff JP, Rosychuk RJ, Joffe AR (2007) The safety and efficacy of sustained inflations as a lung recruitment maneuver in pediatric intensive care unit patients. Intensive Care Med 33:1778-1786

52. von Ungern-Sternberg BS, Hammer J, Frei FJ, Jordi Ritz EM, Schibler A, Erb TO (2007) Prone equals prone? Impact of positioning techniques on respiratory function in anesthetized and paralyzed healthy children. Intensive Care Med 33:1771-1777

53. Santschi M, Gauvin F, Hatzakis G, Lacroix J, Jouvet P (2007) Acceptable respiratory physiologic limits for children during weaning from mechanical ventilation. Intensive Care Med 33:319-325

54. Bruijn $M$, van der Aa LB, van Rijn RR, Bos AP, van Woensel JB (2007) High incidence of acute lung injury in children with Down syndrome. Intensive Care Med 33:2179-2182

55. Albert J, Harbut P, Zielinski S, Ryniak S, Gillis-Haegerstrand C, Lindwall R, Solski L, Lundberg JO, Svensson J, Gozdzik W (2007) Prolonged exposure to inhaled nitric oxide does not affect haemostasis in piglets. Intensive Care Med 33:1594-1601

56. Fassassi M, Michel F, Thomachot L, Nicaise C, Vialet R, Jammes Y, Lagier P, Martin C (2007) Airway humidification with a heat and moisture exchanger in mechanically ventilated neonates : a preliminary evaluation. Intensive Care Med 33:336-343
57. Bollen CW, Uiterwaal CS, van Vught AJ (2007) Meta-regression analysis of high-frequency ventilation vs. conventional ventilation in infant respiratory distress syndrome. Intensive Care Med 33:680-688

58. Capoluongo E, Vento G, Rocchetti S, Giardina E, Concolino P, Sinibaldi C, Santonocito C, Vendettuoli V, Tana M, Tirone C, Zuppi C, Romagnoli C, Novelli G, Giardina B, Ameglio F (2007) Mannose-binding lectin polymorphisms and pulmonary outcome in premature neonates: a pilot study. Intensive Care Med 33:1787-1794

59. Michalek J, Svetlikova P, Fedora M, Klimovic M, Klapacova L, Bartosova D, Elbl L, Hrstkova H, Hubacek JA (2007) Bactericidal permeability increasing protein gene variants in children with sepsis. Intensive Care Med 33:2158-2164

60. Pavcnik-Arnol M, Hojker S, Derganc M (2007) Lipopolysaccharide-binding protein, lipopolysaccharide, and soluble CD14 in sepsis of critically ill neonates and children. Intensive Care Med 33:1025-1032

61. Rey C, Los Arcos M, Concha A, Medina A, Prieto S, Martinez P, Prieto B (2007) Procalcitonin and C-reactive protein as markers of systemic inflammatory response syndrome severity in critically ill children. Intensive Care Med 33:477-484

62. Krueger M, Heinzmann A, Nauck M (2007) Adhesion molecules in pediatric intensive care patients with organ dysfunction syndrome. Intensive Care Med 33:359-363

63. Callister ME, Burke-Gaffney A, Quinlan GJ, Betts H, Nadel S, Evans TW (2007) Persistently low plasma thioredoxin is associated with meningococcal septic shock in children. Intensive Care Med 33:364-367

64. Staquet P, Lemee L, Verdier E, Bonmarchand G, Laudenbach V, Michel C, Lemeland JF, Marret S, Blanc T (2007) Detection of Neisseria meningitidis DNA from skin lesion biopsy using real-time PCR: usefulness in the aetiological diagnosis of purpura fulminans. Intensive Care Med 33:1168-1172

65. Casartelli $\mathrm{CH}$, Garcia PC, Branco RG, Piva JP, Einloft PR, Tasker RC (2007) Adrenal response in children with septic shock. Intensive Care Med 33:1609-1613

66. Garcia-Teresa MA, Casado-Flores J, Delgado Dominguez MA, RoquetaMas J, Cambra-Lasaosa F, ConchaTorre A, Fernandez-Perez C (2007) Infectious complications of percutaneous central venous catheterization in pediatric patients: a Spanish multicenter study. Intensive Care Med 33:466-476
67. Hatherill M, Salie S, Waggie Z, Lawrenson J, Hewitson J, Reynolds L, Argent A (2007) The lactate:pyruvate ratio following open cardiac surgery in children. Intensive Care Med 33:822-829

68. Di Filippo S, Lai Y, Manrique A, Pigula F, Munoz R (2007) Intensive care course after stage 1 Norwood procedure: are there early predictors of failure? Intensive Care Med 33:111-119

69. Bontadelli J, Moeller A, Schmugge M, Schraner T, Kretschmar O, Bauersfeld U, Bernet-Buettiker V, Albisetti M (2007) Enoxaparin therapy for arterial thrombosis in infants with congenital heart disease. Intensive Care Med 33:1978-1984

70. Lemmer J, Stiller B, Heise G, AlexiMeskishvili V, Hubler M, Weng Y, Berger F (2007) Mid-term followup in patients with diaphragmatic plication after surgery for congenital heart disease. Intensive Care Med 33:1985-1992

71. Shankar V, Haque A, Churchwell KB, Russell W (2007) Insulin glargine supplementation during early management phase of diabetic ketoacidosis in children. Intensive Care Med 33:1173-1178

72. Mehta NM, Halwick DR, Dodson BL, Thompson JE, Arnold JH (2007) Potential drug sequestration during extracorporeal membrane oxygenation: results from an ex vivo experiment. Intensive Care Med 33:1018-1024

73. Kneyber MC, Hersi MI, Twisk JW, Markhorst DG, Plotz FB (2007) Red blood cell transfusion in critically ill children is independently associated with increased mortality. Intensive Care Med 33:1414-1422

74. Kuzniewicz M, Draper D, Escobar GJ (2007) Incorporation of physiological trend and interaction effects in neonatal severity of illness scores: an experiment using a variant of the Richardson score. Intensive Care Med 33:1602-1608

75. Wolfler A, Silvani P, Musicco M, Salvo I (2007) Pediatric Index of Mortality 2 score in Italy: a multicenter, prospective, observational study. Intensive Care Med 33:1407-1413

76. Schieveld JN, Leroy PL, van Os J, Nicolai J, Vos GD, Leentjens AF (2007) Pediatric delirium in critical illness: phenomenology, clinical correlates and treatment response in 40 cases in the pediatric intensive care unit. Intensive Care Med 33:1033-1040

77. Cheung CL, van Dijk M, Green JW, Tibboel D, Anand KJ (2007) Effects of low-dose naloxone on opioid therapy in pediatric patients: a retrospective case-control study. Intensive Care Med 33:190-194 